\title{
Giant and multivessel aneurysms of the coronary artery
}

\section{Wael Abuzeid MD, Juan Carlos Monge MD}

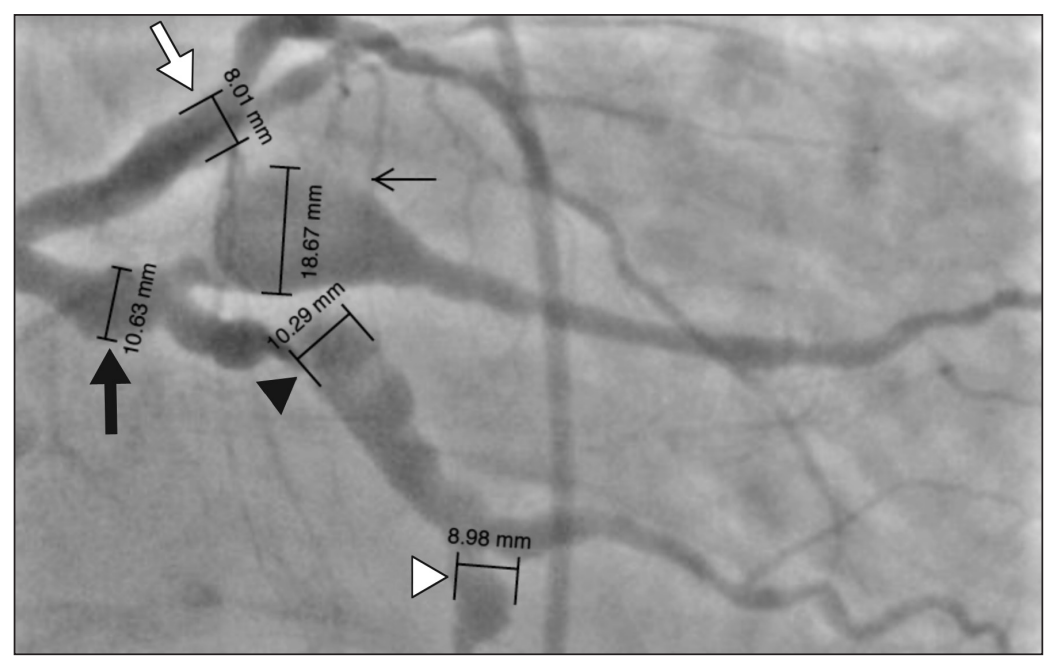

Figure 1: Coronary angiogram showing multiple aneurysms in the proximal left anterior descending artery (white arrow), circumflex artery (proximal, thick black arrow; midlevel, black arrowhead; and distal, white arrowhead) and obtuse marginal branch of the circumflex artery (thin black arrow) in an 82-year-old man with exercise intolerance.

Competing interests: None declared.

This article has been peer reviewed.

The authors have obtained patient consent.

Affiliation: Division of Cardiology, University of Toronto, Toronto, Ont.

Correspondence to: Wael Abuzeid,

wael.abuzeid@mail.utoronto .ca

CMAJ 2015. DOI:10.1503 /cmaj.140702
A n 82-year-old man was referred for cardiac evaluation because of exercise intolerance and atypical chest symptoms (nonexertional intermittent sharp discomfort lasting a few seconds). The patient's medical history included refractory hypertension, myocardial infarction and smoking.

On physical examination, the patient's blood pressure was 165/83 $\mathrm{mm} \mathrm{Hg}$, and his heart rate was 80 beats $/ \mathrm{min}$. A cardiovascular and chest examination were normal except for a highpitched systolic ejection murmur (grade 2 intensity) best heard at the base of the heart.

A persantine sestamibi test showed evidence of ischemia in the distribution of the circumflex branch of the left coronary artery, and a transthoracic echocardiogram showed moderate systolic dysfunction of the left ventricle. Coronary angiography showed an aneurysm in the proximal circumflex coronary artery, a large aneurysm in the first obtuse marginal branch of the circumflex artery (Figure 1) and a giant aneurysm involving the middle segment of the right coronary artery (Figure 2).

Coronary artery aneurysm is defined as a localized dilatation greater than 1.5 times the

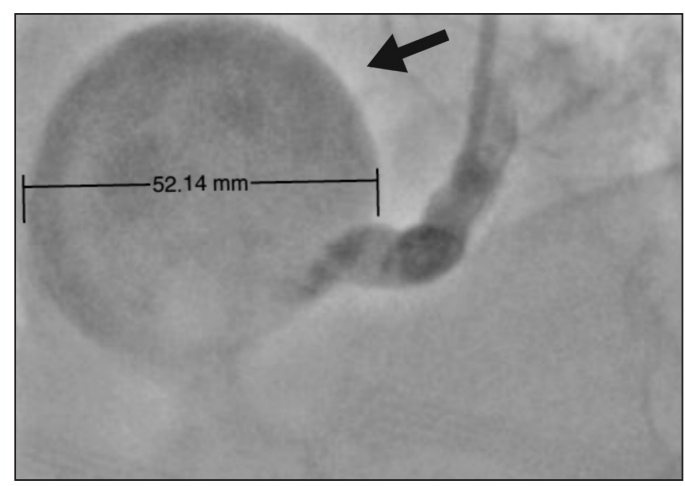

Figure 2: Coronary angiogram showing a giant aneurysm of the right coronary artery (arrow) in an 82-year-old man with exercise intolerance.

diameter of an adjacent segment. ${ }^{2}$ The incidence in the general population ranges from $0.02 \%$ to $0.04 \%$; the incidence in patients undergoing coronary angiography ranges from $1.1 \%$ to $4.9 \%{ }^{1}$ Atherosclerosis is the most common cause $(50 \%$ of cases), followed by Kawasaki disease (10\%$20 \%$ of coronary aneurysms in adults). ${ }^{2}$ Kawasaki disease, a systemic vasculitis of medium and small arteries, is more likely to involve multiple vessels, proximal segments and bifurcations of coronary arteries, and is associated with high early mortality. ${ }^{3}$ Thrombus formation results in $75 \%$ of coronary aneurysms, but reports of rupture are uncommon. ${ }^{1}$

Invasive treatments such as stenting or bypass are reserved for cases of rapid expansion, fistula formation and compression of adjacent structures. Antiplatelet therapy, and in some cases anticoagulant therapy, aims to prevent thromboembolic complications. ${ }^{2}$ Our patient received treatment with acetylsalicylic acid and antihypertensive agents.

\section{References}

1. Keyser A, Hilker MK, Husser O, et al. Giant coronary aneurysms exceeding $5 \mathrm{~cm}$ in size. Interact Cardiovasc Thorac Surg 2012; 15:33-6.

2. Zeb M, McKenzie DB, Scott PA, et al. Treatment of coronary aneurysms with covered stents: a review with illustrated case. J Invasive Cardiol 2012;24:465-9.

3. Rozo JC, Jefferies JL, Eidem BW, et al. Kawasaki disease in the adult: a case report and review of the literature. Tex Heart Inst $J$ 2004;31:160-4 